\title{
Inhalt
}

Heiner Fangerau, Nico Dragano und Stephan Glaremin

Gruß- und Geleitworte - V

Anne Oommen-Halbach, Simone Weyers und Maria Griemmert

Vorwort - XI

Esther Schäfermeier, Laurin Bremerich, Yvonne Gormanns und Alexandru Agache Familienalltag in Zeiten von Corona: Zur Betreuung von Kindern und Stress von Familien -1

Nele Flüchter und Nicole Reese

„Da wird viel Schaden angerichtet“ - Grundschulkinder in der Pandemie — 17

Johanna Luisa Börgermann

Die Schulsituation in der Pandemie aus Sicht der

Landesschüler*innenvertretung NRW - 33

Menno Baumann

Nicht mehr Kind, noch nicht erwachsen: Entwicklungsbedürfnisse Jugendlicher in der Zange der Pandemie — 45

Ulrich Deinet

Kinder und Jugendliche aus Sicht der Einrichtungen der Offenen Kinderund Jugendarbeit: Erste Ergebnisse des Projekts „Neustart der OKJA in NRW“ 53

\section{Christian Nonhoff}

Großwerden in der Pandemie: Vom Baby zum Schulkind aus Sicht der kinderärztlichen Praxis — 63

Simone Weyers

Soziale Ungleichheit in der Kindergesundheit: Status Quo und Hypothesen zur künftigen Entwicklung - 69 
Renate Bredahl

Corona-Pandemie: Entwicklungsprobleme bei Schulneulingen nachweisbar -83

Christine Joisten, Stefanie Wessely und Wanja Noethig

Bewegung im Kindes- und Jugendalter in Zeiten der COVID-19-Pandemie — 91

Birgit Mewes

Möglichkeiten und Grenzen von Erziehungsberatung in der Pandemie. Junge Familien zwischen Resilienz und Resignation — 101

Dominik Wulf

Kinderschutz im Lockdown? Das Childhood-Haus Düsseldorf trotz Corona ein sicherer Ort! - 111

Maria Griemmert

Von Virenschleudern und Partygängern. Die mediale (Re-)Präsentation von Kindern und Jugendlichen in der Pandemie — 119

Anne Oommen-Halbach

Verankerung der Kinderrechte im Grundgesetz? Über die Geschichte und aktuelle Konjunktur einer Debatte —131

Autorenverzeichnis - 145 